\title{
Hands-On Lab Skills Key for Quantum Jobs
}

\section{Quantum companies need physicists who can build qubits and not just integrate wave functions.}

\section{By Katherine Wright}

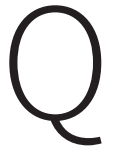
uantum physics courses need updating, according to researchers who interviewed representatives from 21 US companies that specialize in designing and building technologies for quantum applications [1]. The interviews suggest that those graduating with bachelor's degrees in physics may lack the skills they need to enter the quantum workforce. Changing that, the researchers say, could completely alter the face of physics courses and help make the subject relevant to a new generation of students.

"The physics major could become the most sought-after degree for quantum technology companies," says Benjamin Zwickl, a specialist in physics education at the Rochester Institute of

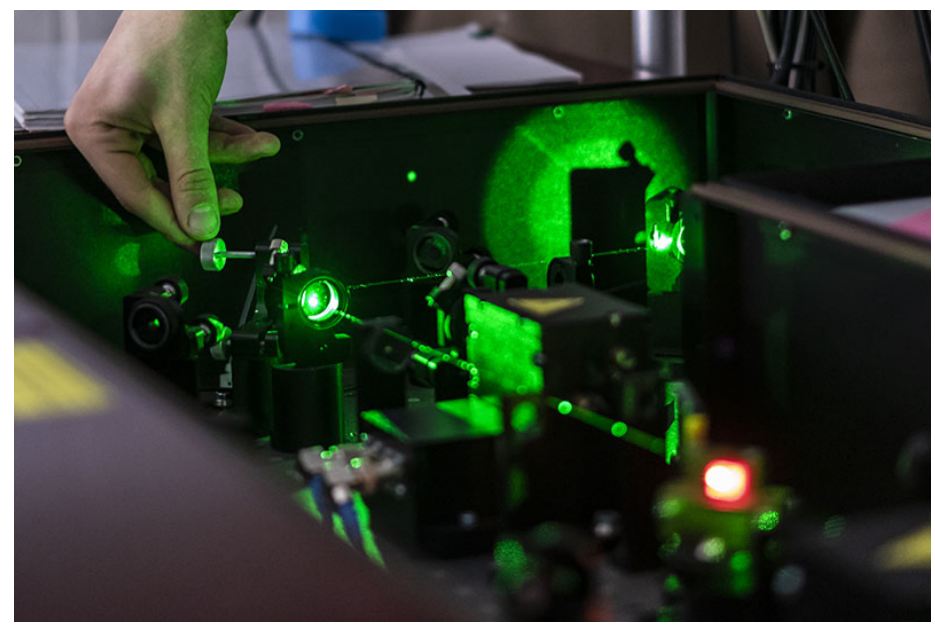

A survey of 21 companies aimed to understand the scientific, technical, and "soft" skills required of new hires into the quantum industry.

Credit: Mihail/stock.adobe.com
Technology in New York and one of the three researchers who conducted the new study.

Once the realm of science fiction, quantum computers are now reality. Companies such as IBM and Google have built functioning quantum machines containing a few tens of qubits and plans are afoot for larger, 1000-qubit computers. To reach that goal, the industry requires some form of quantum-literate workforce, says University of Colorado, Boulder, quantum physicist Heather Lewandowski. She and her colleagues wanted to find out exactly how that quantum literacy is defined by different companies in terms of the skills they require of new hires, so they decided to conduct an industry-wide study.

Most of the companies that the team consulted are part of the Quantum Economic Development Consortium (QED-C), a group working to grow the US quantum industry. The representatives were largely direct supervisors of entry-level technical staff, rather than top-level executives. "We wanted to talk to people who could speak with knowledge about the tasks being carried out in entry-level jobs," Zwickl says.

In the interviews, which each lasted about an hour, the representatives were asked questions aimed at uncovering the scientific, technical, and "soft" skills required of new hires, as well as the needed quantum-specific knowledge. The interviews also covered hiring practices and on-the-job training opportunities.

Analyzing the answers, the team found two common threads. First, companies were often looking for people that were quantum "aware"-they broadly understood the concepts behind quantum computing, sensing, or communication, and 
they could talk about those concepts effectively. The candidates didn't need a deep knowledge of the nitty-gritty equations and theory, however. Second, candidates with hands-on lab skills, such as the ability to make electrical devices, were favored over those with none. "Most of these companies are looking for quantum-literate engineers who can help build quantum devices and make them work reliably," says Michael Fox, who works in physics education research at the University of Colorado, Boulder, and who conducted all of the interviews.

But bachelor-degree graduates with those two skills can be hard to find. Undergraduate physics majors generally have very little experience with building electrical or quantum devices, while engineering undergraduates often have little to no exposure to quantum mechanics, Lewandowski says.

The problem lies in the design of quantum courses. Quantum physics is typically an advanced course, requiring many prerequisites, which can limit access to majors outside of physics, Fox says. The content of the courses is also outdated, focusing on the quantum physics of the early 1900s rather than the "more exciting" advances of the last decade. Another issue is the hands-off format of most introductory quantum classes; laboratory training often comes at the Ph.D. stage for students who specialize in condensed-matter physics or in atomic, molecular, and optical physics. "Improving our undergraduate instruction is really important," Lewandowski agrees. "We need more authentic laboratory experiences and refocused introductory quantum-information courses that have no physics prerequisites."

The trio hope that their study could spur and guide institutions and departments to redesign their courses so that they better address industry needs. Some institutions, including the University of Colorado, Boulder, have started offering "101" quantum information courses, for example, and the team is hopeful that others will follow suit.

These results present the "best assessment so far of the state of the quantum workforce," says Joe Broz, the executive director of the QED-C. He adds that, "studies like this one are critically needed to improve the 'impedance match' between higher education and industry."

Introducing quantum physics at the high-school level could also be a way to bridge the knowledge gap. This idea already has momentum says Denise Ruffner, VP of business development at IonQ in Maryland. She notes that many programs are popping up that are aimed at getting high schoolers interested in careers in the quantum industry, whether as a scientist or engineer or as a marketing or human resources specialist. People can get scared by the word quantum, she says, and as a result they don't send in an application. But, she notes, there are myriad paths to careers in the quantum industry, with only a few requiring that the person be a Ph.D.-level quantum physicist. "Many people working in quantum have very different backgrounds, and that hasn't prohibited them from moving forward in this industry."

Katherine Wright is a Senior Editor for Physics.

\section{REFERENCES}

1. M. F. J. Fox et al., "Preparing for the quantum revolution: What is the role of higher education?" Phys. Rev. Phys. Educ. Res. 16, 020131 (2020). 